\title{
BOUNDARY VALUE PROBLEMS IN PLASMA OSCILLATIONS: THE PLASMA CAPACITOR
}

\author{
F. C. SHURE \\ University of Michigan, Ann Arbor, Mich.
}

(Received 8 July 1963)

\begin{abstract}
A normal mode treatment is proposed for the solution of boundary value problems in plasma oscillations. The plasma distribution function and electric field are expanded in terms of the solutions of the coupled Vlasov and Maxwell equations. These normal mode solutions are somewhat unusual in that they are singular functions of the velocity variable, and, in fact, the expansion theorem is proved by means of a direct solution of an associated singular integral equation. As an application of the method we obtain the impedance of a plasma-filled parallel plate condenser.
\end{abstract}

\section{INTRODUCTION}

A FULLY ionized plasma can support several different kinds of oscillations. The literature on the subject is extensive, and the problems which arise are treated using a wide variety of plasma models.

In this paper we present a method for solving boundary value problems occurring when the plasma is described by the Vlasov 'collisionless' Boltzmann equation. The method involves an expansion in the normal modes of the coupled system of the Vlasov equation and the electromagnetic field equations. (In the example of longitudinal oscillations treated here, only Poisson's equation among the electromagnetic field equations is required.)

In terms of the formal manipulations involved, our treatment is completely straightforward, identical with the normal mode treatment of more familiar types of boundary value problems. However, the normal modes themselves are singular functions, reflecting the singular nature of the streaming operator in the transport equation. This was first recognized by VAN KAMPEN (1955) who constructed a set of fixed-wave number modes for the solution of the initial value problem. CASE (1960) applied the singular normal mode decomposition to boundary value problems in neutron transport theory, and our treatment parallels his to an extent.

In Section 2 we construct the normal modes for fixed-frequency oscillations. It is seen that the eigenvalue spectrum separates into three parts: a pair of discrete imaginary values, a continuum including the entire real axis, and the point at infinity. In Section 3 we construct adjoint modes which, together with the original normal modes, form a biorthogonal set. In Section 4 we prove a completeness theorem for the normal modes by directly solving the singular integral equation implied by the normal mode expansion of an arbitrary function. It is shown that the solution may always be written in terms of the characteristic function $\Lambda(v)$ which has the property that it is analytic in the complex plane cut along the set of points of the continuous spectrum and vanishes only at the discrete eigenvalues. In Sections 5 and 6 we apply the method to the calculation of the electric field within a plasma-filled capacitor and observe the interesting occurrence of the phenomenon of Landau damping governing the resistive impedance of such a device. 


\section{NORMAL MODES FOR LONGITUDINAL OSCILLATIONS}

We consider the problem of obtaining the electric field within a plasma-filled plate condenser upon which an e.m.f. of frequency $\omega$ is imposed. By virtue of the symmetry of the problem* the electric field lies in the $x$-direction (see Fig. 1) and

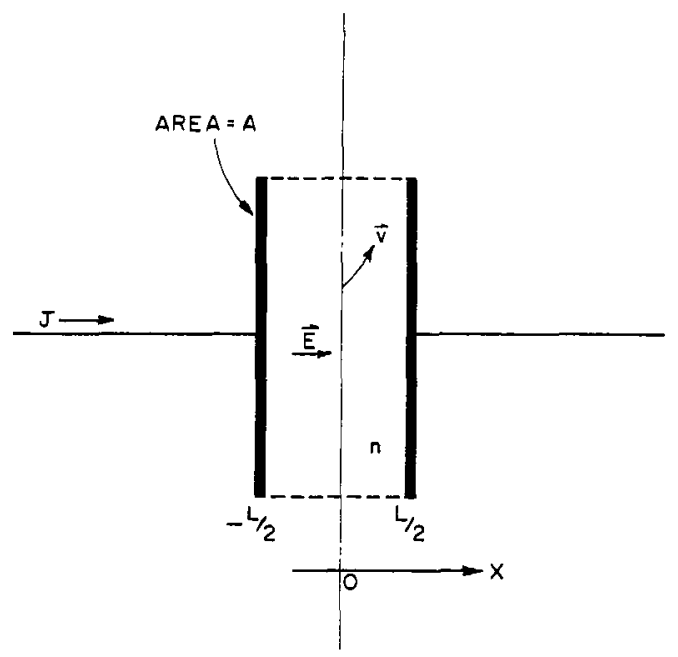

FIG. 1.-Plasma capacitor geometry.

only the $x$-component of the velocity plays a role. The coupled one-dimensional Vlasov and Poisson equations are

$$
\begin{gathered}
\frac{\partial}{\partial t} f(x, u, t)+u \frac{\partial f}{\partial x}=-\frac{n e}{m} E(x, t) \frac{\mathrm{d} F(u)}{\mathrm{d} u} \\
\frac{\partial}{\partial x} E(x, t)=4 \pi e \int_{-\infty}^{\infty} f \mathrm{~d} u .
\end{gathered}
$$

The original Vlasov equation has been linearized about its equilibrium solution

$$
\mathcal{F}(x, u, t)=n F(u)+f(x, u, t)
$$

where $n$ is the electron density and $F(u)$ is a one-dimensional Maxwellian at temperature $\Theta / k$.

In order to emphasize the dependence of the solution on the characteristic plasma parameters, we convert to the non-dimensional length, time and velocity variables

$$
x^{\prime}=\frac{x}{\lambda_{D}} \quad t^{\prime}=t \omega_{n} \quad u^{\prime}=\frac{u}{\lambda_{D} \omega_{p}}
$$

where the Debye length $\lambda_{D}$ and plasma period $\omega_{p}^{-1}$ are given by

$$
\lambda_{D}^{2}=\frac{\Theta}{4 \pi n e^{2}} \quad \omega_{p}^{2}=\frac{4 \pi n e^{2}}{m} .
$$

* In problems involving propagation of transverse waves, the full set of Maxwell's equations is required. However, the treatment is identical in its essentials. 
In addition, the frequency of the impressed field is measured in units of $\omega_{p}$ :

$$
\omega^{\prime}=\omega / \omega_{p} \text {. }
$$

Equations (1) and (2) may then be written entirely in terms of non-dimensional quantities

$$
\begin{gathered}
\frac{\partial f^{\prime}}{\partial t^{\prime}}+u^{\prime} \frac{\partial f^{\prime}}{\partial x^{\prime}}=\frac{-1}{4 \pi} \frac{\mathrm{d} F^{\prime}\left(u^{\prime}\right)}{\mathrm{d} u^{\prime}} E^{\prime} \\
\frac{\partial E^{\prime}}{\partial x^{\prime}}=4 \pi \int_{-\infty}^{\infty} f^{\prime}\left(u^{\prime}, t^{\prime}\right) \mathrm{d} u^{\prime} \\
F^{\prime}\left(u^{\prime}\right)=\frac{1}{\sqrt{2 \pi}} \mathrm{e}^{-u^{\prime 2} / 2} \text { (Maxwell-Boltzmann). }
\end{gathered}
$$

Henceforth we drop the primes.

The system will have time dependence $e^{-i \omega t}$ where we will prescribe wherever necessary that $\omega$ has a small positive imaginary part. This will yield the solution resulting from an applied voltage which has been turned on gradually from its zero value at long times in the past.

It turns out more convenient to write the coupled equations as a single matrix equation

or, symbolically,

$$
\left(\begin{array}{ll}
u & 0 \\
0 & 1
\end{array}\right) \frac{\partial}{\partial x}\left(\begin{array}{l}
f \\
E
\end{array}\right)=\left(\begin{array}{cc}
i \omega & -\frac{1}{4 \pi} \frac{\mathrm{d} F}{\mathrm{~d} u} \\
4 \pi \int_{-\infty}^{\infty} & 0
\end{array}\right)\left(\begin{array}{l}
f \\
E
\end{array}\right)
$$

$$
\rho \frac{\partial \Psi}{\partial x}=H \Psi
$$

where $\Psi$ ' is the 'state vector'

$$
\Psi=\left(\begin{array}{l}
f \\
E
\end{array}\right) .
$$

We immediately obtain three different types of normal mode solutions, the first two with exponential space dependence, the third spatially independent.

Class I : Discrete modes

$$
\begin{aligned}
(\Psi & \left.=\psi_{i} \mathrm{e}^{i \omega x / v_{i}}\right) \\
f_{i} & =\frac{\nu_{i}^{2}}{\omega^{2}} \frac{\mathrm{d} F / \mathrm{d} u}{u-\nu_{i}} \\
E_{i} & =\frac{4 \pi v_{i}}{i \omega} .
\end{aligned}
$$

The eigenvalues $v_{i}$ are the zeros of the characteristic function $\Lambda(\nu)$

$$
\Lambda(\nu)=1-\frac{\nu^{2}}{\omega^{2}} \int_{-\infty}^{\infty} \frac{\mathrm{d} F / \mathrm{d} u}{u-v} \mathrm{~d} \nu .
$$


$\Lambda(\nu)$ is analytic in the complex $\nu$-plane cut along the entire real axis, and tends to a constant value for large $|\nu|$

$$
\Lambda(\infty)=1-\frac{1}{\omega^{2}} .
$$

For Maxwellian $F(u)$, it is easy to show that when $\omega>1$ there are no discrete $\nu_{i}$; when $\omega<1$, there are two: $\nu_{ \pm}= \pm i v_{0}$, with $v_{0}$ real and positive. The value of the discrete root $\nu_{0}$ is shown in Fig. 2.

The physical significance of these discrete modes will be discussed in Section 6 .

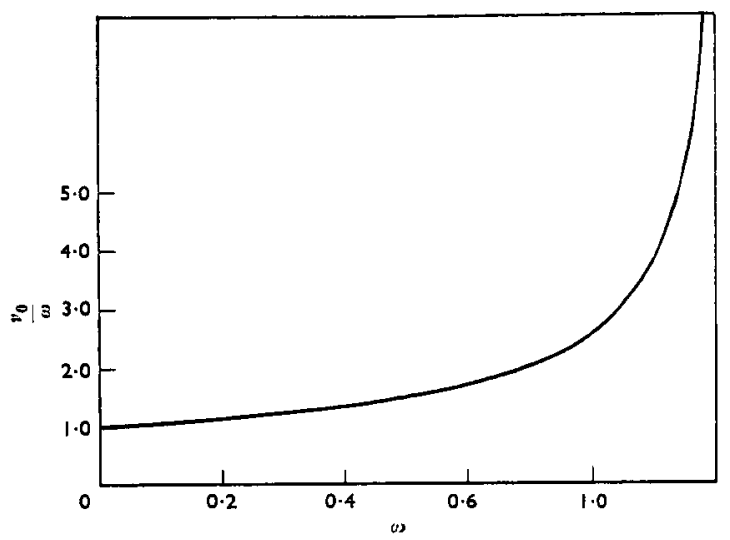

FIG. 2.-Sheath thickness vs. applied frequency (non-dimensionalized).

Class II: Continuum modes

$$
\begin{gathered}
\left(\Psi^{\top}=\psi_{v} e^{i \omega x / v}\right) \quad(\text { all real } v) \\
f_{v}=\frac{\nu^{2}}{\omega^{2}} \mathscr{P} \frac{\mathrm{d} F / \mathrm{d} u}{u-v}+\lambda(\nu) \delta(u-v) \\
E_{v}=\frac{4 \pi v}{i \omega} .
\end{gathered}
$$

Here, the $f_{v}$, are singular functions. $\delta(u-v)$ is the Dirac delta function and the $\mathscr{P}$ indicates the principal value prescription in integrals involving the singular denominator. $\lambda(v)$ is given by

$$
\lambda(v)=1-\frac{v^{2}}{\omega^{2}} \mathscr{P} \int_{-\infty}^{\infty} \frac{\mathrm{d} F / \mathrm{d} u}{u-v} \mathrm{~d} u=\frac{1}{2}\left[\Lambda^{+}(\nu)+\Lambda^{-}(v)\right]
$$

where $\Lambda^{ \pm}(v) \equiv \lim _{\epsilon \rightarrow 0^{+}} \Lambda(\nu \pm i \epsilon)$ are the boundary values of $\Lambda(v)$ as $v$ approaches the cut from above and below respectively. For future reference we note also that

$$
\Lambda^{+}(\nu)-\Lambda^{-}(v)=-2 \pi i \frac{\nu^{2}}{\omega^{2}} \frac{\mathrm{d} F(\nu)}{\mathrm{d} \nu} .
$$


Class III: Charge-free mode

$$
\begin{gathered}
\left(\Psi=\psi_{0}\right) \quad \text { (spatially independent) } \\
f_{0}(u)=\frac{\mathrm{d} F / \mathrm{d} u}{4 \pi i \omega} \\
E_{0}=1
\end{gathered}
$$

These 'fixed-frequency' modes play the same role as the 'fixed-wavenumber' modes constructed by VAN KAMPEN. The necessity for presenting $E$ and $g$ as a twocomponent quantity in our treatment originates in the fact that in the problem of forced oscillations the electric field satisfies a second-order differential equation in the space variable, and it is necessary to specify both $E$ and

$$
\frac{\partial E}{\partial x}\left(\text { i.e. } 4 \pi e \int g \mathrm{~d} u\right),
$$

at $x=0$. In the initial value problem the field satisfies a first-order differential equation in time and one needs work only with the perturbed distribution $g$, since the electric field is related to it simply by

$$
E=\frac{4 \pi e}{i k} \int g \mathrm{~d} u
$$

\section{THE ADJOINT EQUATION AND ORTHOGONALITY RELATIONS}

For every solution of equation (11) there is a solution (with the same value of $v$ ) to the corresponding adjoint equation

$$
\frac{\partial \Psi^{\dagger \dagger}}{\partial Z^{\rho}}=\Psi^{\dagger \dagger} H^{\dagger}
$$

$\Psi^{\dagger}$ is a two-component row vector. The collection of normal modes $\psi_{v}$ and adjoint modes $\psi_{\nu}{ }^{\dagger}$ form a biorthogonal set.

Define a 'scalar product' between normal modes and adjoint modes according to

$$
\left(\psi^{\dagger}, \psi\right)=\int_{-\infty}^{\infty} f^{\dagger}(u) f(u) \mathrm{d} u+E^{\dagger} E .
$$

We then construct the operator $H^{\dagger}$ so that for any $\psi, \psi^{\dagger}$

We find

$$
\left(\psi^{\dagger}, H \psi^{\dagger}\right)=\left(\psi^{\dagger} H^{\dagger}, \psi\right)
$$

$$
H^{\dagger}=\left(\begin{array}{cc}
i \omega & -\frac{1}{4 \pi} \int_{-\infty}^{\infty} \frac{\mathrm{d} F}{\mathrm{~d} u} \\
4 \pi & 0
\end{array}\right) .
$$


Again, the solutions to the adjoint equation fall into three classes:

Class I: Discrete modes

$$
\begin{aligned}
f_{i}^{\dagger}(u) & =\frac{v_{i}^{2} / \omega^{2}}{u-v_{i}} \\
E_{i}^{\dagger} & =\frac{i \nu_{i}}{4 \pi \omega} .
\end{aligned}
$$

As before, the eigenvalues $\nu_{i}$ are just the zeros of $\Lambda(\nu)$.

Class II: Continuum modes (all real $v$ )

with

$$
\begin{gathered}
f_{\nu}^{\dagger}(u)=\frac{v^{2}}{\omega^{2}} \mathscr{P} \frac{1}{u-v}+\lambda^{\dagger}(u) \delta(u-v) \\
E_{\nu}^{\dagger}=\frac{i v}{4 \pi \omega}
\end{gathered}
$$

$$
\lambda^{+}(v)=\frac{\lambda(v)}{\mathrm{d} F(v) / \mathrm{d} v}
$$

Class III: Charge-free mode

$$
\begin{aligned}
f_{0}^{\dagger}(u) & =\frac{4 \pi i}{\omega} \\
E_{0}^{\dagger} & =1 .
\end{aligned}
$$

The orthogonality relations follow immediately from the definition (25) of $H^{\dagger}$. In fact, if

$$
\begin{gathered}
\frac{i \omega}{\nu} \rho \psi_{v}=H \psi_{v} \\
\frac{i \omega}{\nu}{\psi_{v^{\prime}}}^{\dagger} \rho=\psi_{\nu^{\prime}}{ }^{\dagger} H^{\dagger} .
\end{gathered}
$$

Then, by taking the scalar product of (34) and (35) with $\psi_{\nu^{+}}{ }^{\dagger}$ and $\psi_{v}$ respectively and applying (25) we obtain

$$
\frac{i \omega}{v^{\prime}}\left(\psi_{v^{\prime}}{ }^{+}, \rho \psi_{\nu}\right)=\frac{i \omega}{\nu}\left(\psi_{v^{\prime}}{ }^{+}, \rho \psi_{\nu}\right)
$$

or

$$
\left(\psi_{\gamma^{\prime}}{ }^{\dagger}, \rho \psi_{\nu}\right)=0 \quad \nu \neq \nu^{\prime}
$$

This is the orthogonality relation for the modes of Class I and II. To make matters complete, one need only note that

$$
H \psi_{0}=\psi_{0}^{\dagger} H^{\dagger}=0
$$

and apply the same considerations to obtain

$$
\left(\psi_{v}^{\dagger}, \rho \psi_{0}\right)=\left(\psi_{0}^{\dagger}, \rho \psi_{v}\right)=0
$$




\section{THE COMPLETENESS THEOREM}

The orthogonality relations provide a convenient means for performing expansions in terms of the $\psi_{v}$. In this section we prove that the $\left\{\psi_{v}\right\}$ form a complete set: i.e. that such expansions can actually be performed.

First let us note the form that this hypothetical expansion must take. We have

$$
\begin{gathered}
N_{0}=\left(\psi_{0}^{\dagger}, \rho \psi_{0}\right)=\left(1-\frac{1}{\omega^{2}}\right)=\Lambda(\infty) \\
N_{i}=\left(\psi_{i}^{\dagger}, \rho \psi_{i}\right)=\frac{v_{i}^{2}}{\omega^{2}} 1+\frac{\nu_{i}^{2}}{\omega^{2}} \int_{-\infty}^{\infty} \frac{u \mathrm{~d} F / \mathrm{d} u}{\left(u-\nu_{i}\right)^{2}} \mathrm{~d} u \\
=-\left.\frac{\nu_{i}^{3}}{\omega^{2}} \frac{\mathrm{d} \Lambda}{\mathrm{d} \nu}\right|_{\nu=\nu_{i}}=\frac{\nu_{i}^{2}}{\omega^{2}}\left[3-\nu_{i}^{2} \Lambda(\infty)\right] .
\end{gathered}
$$

Moreover, for the continuum modes

and we find

$$
\left(\psi_{v^{\prime}}^{\dagger}, \rho \psi_{v}\right)=N(\nu) \delta\left(\nu-\nu^{\prime}\right)
$$

$$
N(\nu)=\frac{[\lambda(\nu)]^{2}+\left[\pi \frac{\nu^{2}}{\omega^{2}} \frac{\mathrm{d} F(\nu)}{\mathrm{d} v}\right]^{2}}{\left[\frac{\mathrm{d} F(\nu)}{\mathrm{d} \nu}\right]} \nu .
$$

This may also be written in terms of $\Lambda(v)$ by the use of equations (19) and (20).

$$
\frac{1}{N(\nu)}=\frac{\omega^{2}}{2 \pi i \nu^{3}}\left[\frac{1}{\Lambda^{+}(\nu)}-\frac{1}{\Lambda^{-}(\nu)}\right] \text {. }
$$

Therefore, if we assume that a vector $\Psi^{\prime}$ has the expansion

$$
\Psi=A_{0} \psi_{0}+\sum_{i} A_{i} \psi_{i}+\int_{-\infty}^{\infty} A(\nu) \psi_{\nu} \mathrm{d} v,
$$

the coefficients will be determined immediately from the orthogonality relations

$$
A_{0}=\frac{\left(\psi_{0}^{\dagger}, \rho \Psi\right)}{N_{0}} \quad A_{i}=\frac{\left(\psi_{i}^{\dagger}, \rho \Psi\right)}{N_{i}} \quad A(\nu)=\frac{\left(\psi_{\nu}^{\dagger}, \rho \Psi^{p}\right)}{N(\nu)} .
$$

To prove that the expansion is valid we need the following theorem.

Completeness theorem. Any vector $\Psi^{\circ}$ with

$$
\int_{-\infty}^{\infty} f(u) \mathrm{d} u<\infty
$$

may be expanded in terms of the $\left\{\psi_{\nu}\right\}$.

Proof of the theorem:

We have already shown that the expansion coefficients (if they exist) are given by (46). Now let $\Psi^{\circ}$ be an arbitrary vector. If $\Psi$ can be expanded in terms of the $\left\{\psi_{\nu}\right\}$, then $\Psi^{\prime \prime}$ defined by

$$
\Psi^{\prime \prime}=\Psi-A_{0} \psi_{0}-\sum_{i} A_{i} \psi_{i}
$$


with the $A$ 's given by (46), can be expanded in terms of the continuum modes alone:

$$
\Psi^{\prime \prime}=\int_{-\infty}^{\infty} A(\nu) \psi_{v} \mathrm{~d} v
$$

$\Psi^{\prime \prime}$ is orthogonal to the adjoint modes of Classes I and III, but is otherwise arbitrary. Written componentwise this leads to the pair of integral equations for $A(v)$

$$
\begin{gathered}
f^{\prime}(u)=-\frac{1}{\omega^{2}} \frac{\mathrm{d} F(u)}{\mathrm{d} u} \mathscr{P} \int_{-\infty}^{\infty} \frac{\nu^{2} A(\nu) \mathrm{d} \nu}{u-v}+\lambda(u) A(u) \\
E^{\prime}=\frac{4 \pi}{i \omega} \int_{-\infty}^{\infty} \nu A(\nu) \mathrm{d} v .
\end{gathered}
$$

We prove the theorem by obtaining the solution $A(\nu)$ explicitly. Multiplying (50) by

and adding to (49), we obtain:

$$
\frac{i}{4 \pi \omega} \frac{\mathrm{d} F}{\mathrm{~d} u}
$$

$$
u\left[f^{\prime}(u)+\frac{i \mathrm{~d} F / \mathrm{d} u}{4 \pi \omega} E^{\prime}\right]=-\frac{u}{\omega^{2}} \frac{\mathrm{d} F(u)}{\mathrm{d} u} \mathscr{P} \int_{-\infty}^{\infty} \frac{v A(v) \mathrm{d} v}{v-u}+u A(u) \lambda(u) .
$$

Now define the function $\mathscr{A}(v)$ according to

$$
\mathscr{A}(v)=\frac{1}{2 \pi i} \int_{-\infty}^{\infty} \frac{u A(u) \mathrm{d} u}{u-v} .
$$

If $A(\nu)$, the solution to (49) and (50) actually exists, then $\mathscr{A}(\nu)$ will be analytic in the complex plane cut along the real axis and vanish for large $|\nu|$ at least as fast as $|\nu|^{-1}$. The boundary values $\mathscr{A}^{\star}(\nu)$ are related by

$$
\begin{gathered}
\mathscr{A}^{+}(\nu)-\mathscr{A}^{-}(\nu)=v A(\nu) \\
\mathscr{A}^{+}(\nu)+\mathscr{A}^{-}(v)=\frac{1}{\pi i} \mathscr{P} \int_{-\infty}^{\infty} \frac{u A(u) \mathrm{d} u}{u-\nu} .
\end{gathered}
$$

Conversely, if $\mathscr{A}(\nu)$ possessing the required analyticity properties can be found, then $A(\nu)$ will exist and be given by (53).

By the use of the relations (19-20) and (53-54) equation (51) simplifies to

$$
u\left[f^{\prime}(u)+\frac{i \mathrm{~d} F / \mathrm{d} u}{4 \pi \omega} E^{\prime}\right]=\Lambda^{+}(v) \mathscr{A}^{+}(v)-\Lambda^{-}(v) \mathscr{A}-(v) .
$$

Now consider the function $K(v)$ defined by

$$
K(\nu)=\frac{1}{2 \pi i} \int_{-\infty}^{\infty} \frac{f^{\prime}(u)+\frac{i \mathrm{~d} F / \mathrm{d} u}{4 \pi \omega} E^{\prime}}{u-\nu} u \mathrm{~d} u .
$$


$K(v)$ is constructed so that the difference $K^{+}(v)=K^{-}(v)$ is equal to the left-hand side of (55). Hence

$$
\Lambda^{+}(\nu) \mathscr{A}^{+}(\nu)-K^{+}(v)=\Lambda^{-}(\nu) \mathscr{A}^{-}(v)-K^{-}(v)
$$

i.e. the function $\Lambda(v) \mathscr{A}(v)-K(v)$, analytic in the cut plane, is also continuous across the cut. This means that $\Lambda(v) \mathscr{A}(v)-K(v)$ is an entire function and since $K(\nu)$ and $\mathscr{A}(\nu)$ both must vanish for large $|v|$, it follows from Liouville's theorem that

$$
\Lambda(v) \mathscr{A}(v)-K(v)=0
$$

or

$$
\mathscr{A}(\nu)=\frac{K(\nu)}{\Lambda(v)} .
$$

We must examine the function $\mathscr{A}(\nu)$ to see if it has the properties it was assumed to have. We note immediately that $\mathscr{A}(\nu)$ is analytic in the cut plane except possibly at the points $\nu_{i}$ where the denominator vanishes. Thus, for (51) to possess a solution it is necessary and sufficient that

$$
K\left(\nu_{i}\right)=0 .
$$

But we have already insured that this condition be fulfilled by requiring that $\Psi^{\prime \prime}$ be orthogonal to the discrete adjoint modes. In fact $K\left(\boldsymbol{v}_{i}\right)$ is easily found to be given by

$$
K\left(v_{i}\right)=\frac{\omega^{2}}{v_{i}^{2}} \frac{1}{2 \pi i}\left(\psi_{i}^{\dagger}, \rho \Psi^{\prime \prime}\right)=0 .
$$

Finally we must show that the $A(v)$ obtained as a solution of equation (51) actually satisfies $(50)$. Since

$$
\int_{-\infty}^{\infty} \nu A(v) \mathrm{d} v=-\lim _{|z| \rightarrow \infty} 2 \pi i z \mathscr{A}(z)
$$

this last requirement becomes

$$
E^{\prime}=\frac{4 \pi}{i \omega} \frac{1}{\Lambda(\infty)} \int_{-\infty}^{\infty}\left[f^{\prime}(u)+\frac{i \mathrm{~d} F / \mathrm{d} u}{4 \pi \omega} E^{\prime}\right] u \mathrm{~d} u
$$

which becomes, on rearranging

$$
\left(\psi_{0}^{\dagger}, \rho \Psi^{\prime \prime}\right)=0 .
$$

But this is just the last condition that we placed on the function $\Psi^{\prime \prime}$ at the start.

This completes the proof of the theorem.

\section{SOLUTION FOR THE PLASMA CAPACITOR}

So far, everything has been quite general. We have exhibited a complete set of normal modes for longitudinal plasma oscillations. The solution to a given problem is obtained via the normal mode expansion in much the same manner as the solution to more familiar (i.e. non-singular) boundary value problems. One expands the state function of the system in terms of the normal modes and applies the boundary conditions to determine the expansion coefficients, usually with the help of orthogonality relations obeyed by the normal modes. Now we apply the method to a specific problem. 
We assume the plasma to be uniformly distributed between two infinite, perfectly reflecting walls, i.e. a plasma capacitor (see Fig. 1). This is the problem treated by WEISSGLAS (1962) by an alternative method. Before commenting on WEISSGLAS' treatment, let us obtain the complete solution via the normal mode expansion.

The assumption that electrons are specularly reflected at each plate $(x= \pm L / 2)$ implies the boundary condition

$$
f(u, \pm L / 2)=f(-u, \pm L / 2) .
$$

This is sufficient to obtain the entire state function in terms of the electric field across the plates. Write:

$$
\Psi=A_{0} \psi_{0}+A_{+} \mathrm{e}^{\omega x / \nu_{0}} \psi_{+}+A_{-} \mathrm{e}^{-\omega x / \nu_{0}} \psi_{-}+\int_{-\infty}^{\infty} A(\nu) \mathrm{e}^{i \omega x / \nu} \psi_{\nu} \mathrm{d} \nu .
$$

$\pm i v_{0}$ are the two zeros of the characteristic function $\Lambda(v)$. It is understood that the terms with $\psi_{+}$and $\psi_{-}$are to be included only when $\omega<1$. Now we make use of the symmetry properties of the normal modes:

$$
\begin{gathered}
\text { Continuum }\left\{\begin{array}{r}
f_{v}(-u)=f_{-\vartheta}(u) \\
E_{v}=-E_{-v}
\end{array}\right. \\
\text { Discrete }\left\{\begin{array}{r}
f_{+}(-u)=f_{-}(u) \\
E_{+}=-E_{-}
\end{array}\right. \\
\text {Charge-free } f_{0}(u)=-f_{0}(-u) .
\end{gathered}
$$

so that condition $(65+)$ becomes

where

$$
B_{0} f_{0}(u)+B_{+} f_{+}(u)+B_{-} f_{-}(u)+\int_{-\infty}^{\infty} B(\nu) f_{\nu}(u) \mathrm{d} v=0
$$

$$
\begin{aligned}
B_{0} & =2 A_{0} \\
B_{ \pm} & = \pm\left[A_{+} \mathrm{e}^{\omega L / 2 v_{0}}-A_{-} \mathrm{e}^{-\omega L / 2 v_{0}}\right] \\
B(v) & =A(v) \mathrm{e}^{i \omega L / 2 v}-A(-v) \mathrm{e}^{-i \omega L / 2 v} .
\end{aligned}
$$

Moreover, we see from (68) and (70)

$$
B_{0} E_{0}+B_{+} E_{+}+B_{-} E_{-}+\int_{-\infty}^{\infty} B(\nu) E_{v} \mathrm{~d} v=2 E(L / 2) .
$$

Hence the $B$ 's are just the coefficients in the expansion of the vector

$$
\Phi=\left(\begin{array}{c}
0 \\
2 E(L / 2)
\end{array}\right)
$$

and may be obtained immediately from the orthogonality relation: e.g.

$$
B_{0}=\frac{\left(\psi_{0}^{\dagger}, \rho \Phi\right)}{N_{0}}=2 E(L / 2) \frac{E_{0}^{\dagger}}{N_{0}}
$$

and similarly for the rest. 
Applying the same considerations to the plate at $x=-L / 2$ and combining, we obtain the values for all the expansion coefficients

$$
\begin{gathered}
A_{0}=\frac{E(L / 2)}{N_{0}}=\frac{E(-L / 2)}{N_{0}} \\
A(\nu)=E(L / 2) \frac{E_{v}^{\dagger}}{N(v)} \frac{1}{\cos \left(\frac{\omega}{\nu} \frac{L}{2}\right)} \\
A_{\perp}=E(L / 2) \frac{E_{ \pm}^{\dagger}}{N_{ \pm}}-\frac{1}{\cosh \left(\frac{\omega}{v_{0}} \frac{L}{2}\right)} .
\end{gathered}
$$

These results substituted into equation (66) yield the complete formal solution to the problem.

\section{ANALYSIS OF THE SOLUTION}

Just as in the completeness proof of Section 4 we shall rely heavily on the analyticity properties of the characteristic function $\Lambda(v)$ in order to place our solution in a form suitable for computation. We are able to do this since all the $N$ 's of equations (40-44) are simple functions of $\Lambda(\nu)$ and its boundary values of $\Lambda^{ \pm}(\nu)$.

Consider the expression (66) for the electric field between the plates

Since, from (44)

$$
\begin{aligned}
E(x)=E(L / 2) & \left\{\frac{1}{N_{0}}-\frac{\left(v_{0} / \omega\right)^{2}}{N_{+}} \frac{\mathrm{e}^{\omega x / v_{0}}}{\cosh \left(\frac{\omega}{v_{0}} \frac{L}{2}\right)}-\frac{\left(v_{0} / \omega\right)^{2}}{N_{-}} \frac{\mathrm{e}^{-\omega x / v_{0}}}{\cosh \left(\frac{\omega}{v_{0}} \frac{L}{2}\right)}\right. \\
& \left.+\lim _{R \rightarrow 0} \int_{-R}^{R} \frac{(v / \omega)^{2} \mathrm{e}^{i \omega x / v}}{N(v) \cos \left(\frac{\omega}{v} \frac{L}{2}\right)} \mathrm{d} v\right\} .
\end{aligned}
$$

$$
\frac{(\nu / \omega)^{2}}{N(\nu)}=\frac{1}{2 \pi i} \frac{1}{\nu}\left[\frac{1}{\Lambda^{+}(\nu)}-1\right]-\frac{1}{2 \pi i} \frac{1}{\nu}\left[\frac{1}{\Lambda^{-(\nu)}}-1\right]
$$

the integrand in (80) may be broken up into two terms which may be analytically continued into the upper and lower half-planes respectively. Thus the integral term in (80) is given by

$$
\begin{aligned}
\mathscr{I}=\frac{1}{2 \pi i} \int_{C_{1}} \frac{\mathrm{e}^{i \omega x / \nu}}{\cos \left(\frac{\omega}{v} \frac{L}{2}\right)}\left[\frac{1}{\Lambda(\nu)}-1\right] \frac{\mathrm{d} \nu}{v} & \\
& -\frac{1}{2 \pi i} \int_{C_{2}} \frac{\mathrm{e}^{i \omega x / v}}{\cos \left(\frac{\omega}{\nu} \frac{L}{2}\right)}\left[\frac{1}{\Lambda(\nu)}-1\right] \frac{\mathrm{d} \nu}{\nu} .
\end{aligned}
$$

The curves $C_{1}$ and $C_{2}$ (see Fig. 3) are each semicircles of radius $R$ along with small 
circles about the singularities of the integrand in the upper and lower half-planes respectively. These singularities lie at

$$
\nu=i v_{0} \quad \text { and } \frac{\omega}{(2 n+1) \pi} \quad n=0,1,2, \ldots
$$

in the upper half-plane and

$$
\nu=-i v_{0} \text { and } \frac{-\omega L}{(2 n+1) \pi} \quad n=0,1,2, \ldots
$$

in the lower half-plane. It is easily seen from equation (41) that the residue contributions from the poles at $\pm i v_{0}$ identically cancel the second and third terms in (80).

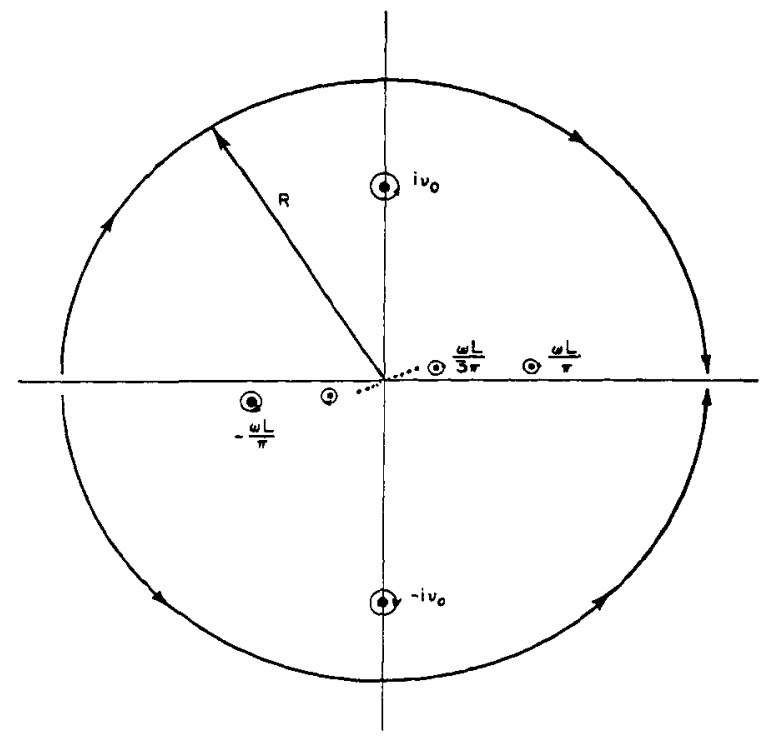

FIG. 3.--Deformed contours for the continuum integral. $C_{1}$ lies wholly in the upper half-plane; $C_{2}$ in the lower.

The integrals along the large semicircles yield a contribution of $[1-1 / \Lambda(\infty)]$ which combines with the first term in (80). This together with the contributions from the infinite sequence of poles close to the real axis yields

$$
E(x)=E(L / 2)\left\{1+\frac{4}{\pi} \sum_{\substack{n=\\ 1,3,5}} \frac{(-1)^{(n-1) / 2}}{n}\left[\frac{1}{\Lambda^{+}\left(\frac{\omega L}{n \pi}\right)}-1\right] \cos (n \pi x / L)\right\} .
$$

It should be mentioned that for low frequencies this representation is inconvenient since the series converges too slowly. Returning then to the original form (80) of the solution, we find that when $\omega \approx 3 / 4$ the contribution from the discrete modes dominates. This is of the form

$$
\sim \cosh \left[\frac{\omega}{v_{0}} x\right] / \cosh \left[\frac{\omega}{v_{0}} \frac{L}{2}\right]
$$


and represents a field which falls off practically to zero in a short distance from the plates. The shielding length $v_{0} / \omega$ is shown in Fig. 2 and is seen to be a slowly varying function of $\omega$, except as $\omega \rightarrow 1$.

The impedance $Z$ of the capacitor is found from Ohm's law

But from (85)

$$
Z J=\int_{-L / 2}^{L / 2} E(x) \mathrm{d} x
$$

$$
\int_{-L / 2}^{L / 2} E(x) \mathrm{d} x=E(L / 2) L \frac{8}{\pi^{2}} \sum_{\substack{n=\overline{1} \\ 1,3,5, \ldots \Lambda^{+}\left(\frac{\omega L}{n \pi}\right)}} \frac{1}{n^{2}}
$$

and $J$ is given by the displacement current at the plates since there is no electron transport across the boundary of the plasma

$$
\frac{J}{A}=\frac{i \omega}{4 \pi} E(L / 2)
$$

$A$ is the area of the plates. Hence

with

$$
Z=\frac{1}{i \omega C_{0}} \frac{1}{\epsilon_{\mathrm{eff}}}
$$

$$
C_{0}=\frac{A}{4 \pi L}
$$

the capacitance of condenser without the plasma, and with

$$
\epsilon_{\mathrm{eff}}=\left[\frac{8}{\pi^{2}} \sum_{\substack{n= \pm 1,3,5 \ldots}} \frac{1}{n^{2}} \frac{1}{\Lambda^{+}\left(\frac{\omega L}{n \pi}\right)}\right]^{-1}
$$

the effective dielectric coefficient.

Another way to interpret these results is to regard the plasma capacitor as a series array of separate devices, each with impedance

$$
Z_{n}=\frac{1}{i \omega C_{0}} \frac{8}{\pi^{2}} \frac{1}{n^{2} \Lambda^{+}\left(\frac{\omega L}{n \pi}\right)} \quad n=1,3,5 \ldots
$$

For most frequencies, $Z=\Sigma Z_{n}$ will be purely reactive since $\operatorname{Im} \Lambda^{+} \ll \operatorname{Re} \Lambda^{+}$. Typically, $L \sim 10^{4}$ and we will almost always be able to approximate $\Lambda^{+}$by $\Lambda(\infty)$ to obtain the familiar result:

$$
\mathcal{Z}=\frac{1}{i \omega C_{0}} \frac{1}{1-\frac{1}{\omega^{2}}} .
$$

However, at the 'resonant' frequencies $\omega_{n}$ for which

$$
\operatorname{Re} \Lambda^{+}\left(\frac{\omega_{n} L}{n \pi}\right)=0
$$


the impedance takes on a significant resistive component and the capacitor becomes a dissipative device. This is effectively WeIssGLAs' explanation.

The interesting point is that the resonant frequencies are just those frequencies for which, in Landau's (1946) theory, a longitudinal wave with wavelength given by

$$
\lambda_{n}=\frac{2 L}{n} \quad n=1,3,5 \ldots
$$

can propagate. Furthermore, the rate of absorption at a resonance is just given by the damping decrement in the Landau theory.

It is worthwhile to reassert that there should be no objection to the procedure of solving the collisionless equation by the device of assuming that $\omega$ has a positive imaginary part which is later allowed to tend to zero. This is, in actuality, a reasonable representation of the time dependence of a system which builds up to its steady state $\sim \mathrm{e}^{-i \omega t}$ over a long period of time. WeISSGLAs' objection that there is actually no steady state for the collisionless equation is quite correct. In fact, if one uses equations (77-79) to obtain the actual distribution function $f(u)$, it turns out that the result is singular at precisely the velocities $\omega L / n \pi$ with which an electron may make a round trip in an odd number of periods. In the collisionless theory of DAwson (1961), the distribution function in the neighbourhood of these velocities does, in fact, increase in time without bound. This is simply an illustration of the fact that Landau damping can serve as a mechanism to transfer electric energy into particle motions within the framework of the collisionless theory in which no entropy increase can take place. Hence some truly dissipative mechanism must be present in addition. However, this does not invalidate the solution, but only places limits on its validity: namely that the collisional mechanism that randomizes the electrons that are bunched into beams via the Landau damping process must be strong enough to forestall the violation of the linearization assumption. This is not a severe restriction. Moreover, the admissible collisional mechanism is quite arbitrary, and does not need to take on the phenomenological form prescribed by WeIssGLAS. In any event, it is interesting that the resistive properties of the plasma capacitor should be governed by the non-dissipative process of Landau damping.

Acknowledgment-The author would like to thank K. M. CASE for his helpful and stimulating comments.

\section{REFERENCES}

CASE K. M. (1960) Ann. Phys. (N.Y.) 9, 1.

DAwSON J. (1961) Phys. Fluids 4, 869.

Kampen N. G. Van (1955) Physica 21, 949.

Werssglas P. (1962) Plasma Phys. (J. Nucl. Energy Part C) 4, 329. 